\title{
A RAPID SINGLE-STEP SCREENING METHOD FOR GLUCOSE-6-PHOSPHATE DEHYDROGENASE DEFICIENCY IN FIELD APPLICATIONS
}

\author{
KUNI IWAI ${ }^{1}$, HIROYUKI MATSUOKA ${ }^{1}$, FUMIHIKO KAWAMOTO², MEIJI ARAI ${ }^{1}$, \\ SHIGETO YOSHIDA ${ }^{1}$, MAKOTO HIRAI $^{1}$ and AKIRA ISHII ${ }^{1}$ \\ Accepted 25, June, 2003
}

\begin{abstract}
The single-step screening method (SSS) is a qualitative rapid screening test for glucose-6-phosphate dehydrogenase (G6PD) deficiency based on blue formazan formation on anion-exchanger. The reaction mixture contains equal volumes of anion exchanger, substrate mixture, coloring mixture (MTT-PMS mix) and distilled water. We assessed the stability of the reaction mixture and evaluated its reliability with two anion exchangers, DEAESephadex A- $50^{\mathrm{TM}}$ and DEAE-Sephacel ${ }^{\mathrm{TM}}$, for applications in tests under field conditions. The reaction mixture was sufficiently stable under conditions of incubation at $70^{\circ} \mathrm{C}$ for 6 hours or vigorous shaking for 24 hours at room temperature. The reaction mixture could be kept at $30-35^{\circ} \mathrm{C}$ for 14 days under indoor conditions without shielding if it contained no MTT-PMS mix. The coloring was detectable even in diluted blood with hemoglobin concentration as low as $1.6 \mathrm{~g} / \mathrm{dl}$. Under laboratory conditions, the proportion of the samples with $10 \%$ of the normal level of activity that were diagnosed as 'low activity' was higher with DEAE-Sephacel (92\%) than with DEAE-Sephadex A-50 $(81 \%)(\mathrm{p}=0.023)$. The proportion of the samples with normal activity that were diagnosed as 'normal' was $98 \%$ with DEAE-Sephacel and 100\% with DEAE-Sephadex A-50. In field samples obtained in Myanmar and Indonesia, the sensitivity was lower ( $\mathrm{P}=0.03$ using DEAE-Sephadex and $\mathrm{p}<0.001$ using DEAE-Sephacel) when we used the blue formazan spot test (BFST) as the standard. Twenty-three of 27 G6PD-deficient individuals subjected to genetic analysis were found to have mutations. All individuals who had concordant results between the SSS and the blue formazan spot test (BFST) carried molecular mutations. One case of G6PD mutation was detected among four cases diagnosed as G6PD-deficient by SSS with DEAE-Sephacel ${ }^{\mathrm{TM}}$, but diagnosed as 'normal' by BFST. The costs of one test with the DEAE-Sephadex A- $50^{\mathrm{TM}}$ and the DEAE-Sephacel ${ }^{\mathrm{TM}}$ system were 0.15 US dollar and 0.30 US dollar, respectively. (297 words)
\end{abstract}

Key words: Single-step screening test, Glucose-6-phosphate dehydrogenase deficiency, Device approval, Field trial, Malaria

\section{INTRODUCTION}

Glucose-6-phosphate dehydrogenase (G6PD) deficiency is a frequent and heterogeneous $\mathrm{X}$-chromosomelinked enzyme abnormality. As G6PD plays a key role in maintaining erythrocytes, G6PD deficiency possibly results in acute hemolysis after exposure to various oxidative stresses, including infections, medications, and fava beans (favism). A striking correlation is observed between the prevalence of G6PD deficiency and historical malaria endemicity, particularly in tropical and sub-tropical areas (W. H.O. Working Group, 1989). Therefore, it is important to detect and inform G6PD-deficient individuals in and from areas in which malaria is endemic before exposing such individuals to oxidative stress in order to avoid acute hemo- lytic attack, especially hemolytic attack caused by primaquine.

For field screening of G6PD deficiency, the test used should be simple to perform and affordable. It is also advantageous if the test reagents can be stored and the reaction can be carried out at around room temperature, particularly in areas with an insufficient supply of electricity.

Several tests, including the G6PD spot test (Fairbanks and Beutler, 1962), fluorescent spot test (Beutler, 1966), and blue formazan spot test (Fujii et al., 1984) have been developed for field screening of G6PD deficiency; however most of them are complicated or expensive. One of the tests, the blue formazan spot test (BFST) (Fujii et al ., 1984; Pujades et al., 1999), has been used for field studies because of its comparatively simple procedure and sufficiently 
low cost. However, BFST requires 8 hours of incubation for diagnosis, which makes immediate on-site diagnosis difficult.

Recently, a simple and quick screening method, a single-step screening test (SSS) was developed (Hirono et al., 1998). Our previous trials showed the usefulness of SSS in field studies (Tantular et al., 1999), but the stability and reliability of the test have not been fully assessed. In this study, we assessed in detail the stability of SSS and the results of the reliability of SSS use under field conditions.

\section{MATERIALS AND METHODS}

SSS is a qualitative test that assesses the formation of blue formazan along with the reduced form of nicotinamide adenine dinucleotide phosphate (NADPH) by G6PD absorbed on an anion exchanger. The reaction mixture consists of equal volumes (200 $\mu \mathrm{l}$ each) of substrate mixture containing $5 \mathrm{mM}$ glucose-6-phosphate (G6P) and $0.4 \mathrm{mM}$ oxidized nicotinamide adenine dinucleotide phosphate (NADP), coloring mixture (MTT-PMS mix) containing $0.025 \% 3$ (4,5 dimethylthiazolyl 1-2) 2,5 diphenyltetrazolium bromide (MTT), 0.025\% phenazine methosulphate (PMS), anion exchanger (originally DEAE-Sephadex A$50^{\mathrm{TM}}$ ) and distilled water. The test can detect G6PDdeficient individuals with less than $10 \%$ residual activity within 40 minutes without any special equipment.

The procedures and chemicals used for SSS (Hirono et al ., 1998) and BFST (Fujii et al ., 1989) were as described, with slight modifications in the procedure for SSS: $0.05 \%$ sodium azide $\left(\mathrm{NaN}_{3}\right)$ was added and saponin was omitted in the reaction mixture to avoid the growth of microorganisms. We assessed the reliability of two different anion exchangers in SSS: translucent DEAE-Sephadex A-50 ${ }^{\mathrm{TM}}$ and whitecolored DEAE-Sephacel $^{\mathrm{TM}}$ (Amersham Pharmacia Biotech, Buckinghamshire, UK). When using the DEAE-Sephacel ${ }^{\mathrm{TM}}$ system, $4.8 \mathrm{mM}$ of oxidative glutathione (GSSG) was added to optimize the reactivity (Hirono et al ., 1998).

The blood samples for laboratory assessment were drawn from one of the authors who was confirmed phenotypically and genotypically to have normal G6PD. The G6PD gene was analyzed by direct sequencing of all coding exons. The G6PD-deficient blood was artificially made from the blood of the same volunteer by heat inactivation at $56^{\circ} \mathrm{C}$ for 15 minutes and proved to have no residual G6PD activity. Nine volumes of heat-inactivated blood and one volume of fresh blood were mixed to make G6PD-deficient samples with $10 \%$ of the normal activity. The samples for field comparison and genetic analysis were obtained from local residents who participated in an extensive field study with the aim of quick detection of malaria and G6PD defi- ciency (Tantular et al. 1999) in the Taninthayi Division in Myanmar and on Buru Island in Indonesia, 1998. Prior to the study, the outline and procedures of this research were discussed within the committees of the national and local governments in Indonesia and Myanmar. The participants were orally informed of and gave consent to the examination twice before screening and blood drawing. We did not obtain written informed consent from each participant because of the opinion of local co-organizers about the participants' literacy and differences of local culture. The field samples were used to assess the sensitivity and specificity of SSS using BFST as the standard. We also compared the 'G6PD-deficient' samples of SSS with BFST, using genetic analysis as the standard.

To assess the stability of the test mixtures of SSS under various laboratory conditions, we simulated three different conditions which may often occur during field studies in tropical areas: $30-35^{\circ} \mathrm{C}$ for 14 days (storage at room temperature), $70^{\circ} \mathrm{C}$ for 6 hours in an oven (being left in a car), continuous shaking using a shaker (TAITEC R-1) at maximum strength (200 rotations/minute) for 24 hours at room temperature (transporting over a rough surface). We also examined the photosensitivity of the test, because the dissolved MTT-PMS mixture is highly photosensitive (Fairbanks and Beutler, 1962). For simulating anemia, which is a common illness and may affect the results (Fairbanks and Beutler, 1962), serial two-fold dilutions of blood with normal G6PD activity from the same volunteer in other laboratory tests were made with phosphate buffered saline (PBS) to one-eighth of the original concentration (the estimated hemoglobin concentration was then $1.6 \mathrm{~g} / \mathrm{dl}$ ). For all of the assessments, the combination of newly prepared testing mixture and fresh blood was used for the standard of the normal reaction.

For the evaluation of reliability, the results were categorized into two groups, 'low activity' and 'normal activity', based on color development of the anion-exchanger. Two investigators who were blinded to the actual activity of samples then evaluated the enzyme activity (50 deficient and 50 normal per person) independently as 'low activity' or 'normal activity'. The results were analyzed by the chi-square test using a $2 \times 2$ table or Fisher's exact test. P values less than 0.05 were considered as statistically significant.

BFST was used as the standard for field assessment of the sensitivity and specificity of SSS. The SSS was performed at each field site and samples for BFST were obtained on cation-exchange paper (Whatman P-81) (Fujii et al ., 1984) at the same time and dried. The BFST was performed within the same day, and thus the results were obtained the next day. The results were analyzed statistically as described above. 
For comparison of the results of SSS and BFST with the results of genetic analysis, the participants were screened by SSS, and a blood spot was obtained in the same way as described above. The participants were diagnosed with G6PD deficiency based on the results of SSS, and their blood was drawn for genetic analysis. The procedures for genetic analysis were described previously (Hirono et al ., 1994; Hirono et al ., 1997, Iwai et al ., 2001).

\section{RESULTS AND DISCUSSION}

The reaction mixture of SSS retained sufficient reactivity compared with the control mixture after exposure to $70^{\circ} \mathrm{C}$ for 6 hours or continuous shaking for 24 hours at room temperature. Reaction mixture without the MTT-PMS mix could be stored at $30-35^{\circ} \mathrm{C}$ for 14 days under indoor conditions without shielding. These features would make this test appropriate for regular use in primary care and in field studies in rural areas in developing countries. A previously reported 1-year storage trial (Hirono et al., 1998) showed that DEAE-Sephadex ${ }^{\mathrm{TM}}$ can be kept at room temperature, the MTT-PMS mix can be stored at $4^{\circ} \mathrm{C}$, and the substrate mixture is stable at $-20^{\circ} \mathrm{C}$. If a refrigerator and freezer are available, each mixture can be safely stored for at least1year.

In our simulation of anemia, the reaction mixture yielded detectable color development when the estimated hemoglobin concentration of the G6PD-normal blood was as low as $1.6 \mathrm{~g} / \mathrm{dl}$. Because elevated levels of reticulocytes lead to higher activity of peripheral blood G6PD in the anemic condition (Jansen et al ., 1985), our finding implies that the G6PD-normal samples of patients with severe anemia should not be frequently misdiagnosed as 'G6PD-deficient'.

Table 1 shows the reliability of the 2 anion-exchangers. Under laboratory conditions, a higher proportion of the samples with $10 \%$ of normal activity were diagnosed as

Table 1. Laboratory evaluation of the single-step screening method (SSS) with 2 anion exchangers

A. DEAE-Sephadex A-50

\begin{tabular}{cccc}
\hline Diagnosis & \multicolumn{2}{c}{ Residual activity of G6PD } & \multirow{2}{*}{ Total } \\
\cline { 2 - 3 } by SSS & $10 \% *$ & $100 \%$ & \\
\hline Low activity & 81 & 0 & 81 \\
Normal activity & 19 & 100 & 119 \\
\hline Total & 100 & 100 & 200 \\
\hline
\end{tabular}

\begin{tabular}{cccc} 
B. DEAE-Sephacel & & & \\
\hline & \multicolumn{2}{c}{ Residual activity of G6PD } & Total \\
\cline { 2 - 3 } by SSS & $10 \%^{*}$ & $100 \%$ & \\
\hline Low activity & 92 & 2 & 94 \\
Normal activity & 8 & 98 & 106 \\
\hline Total & 100 & 100 & 200 \\
\hline
\end{tabular}

*The proportion is significantly different between the DEAE-Sephadex A-50 system and DEAE-Sephacel system 'low activity' with DEAE-Sephacel ${ }^{\mathrm{TM}}$ (92\%) than with DEAE-Sephadex A-50 $0^{\mathrm{TM}}(81 \%) \quad(\mathrm{p}=0.023)$. With the DEAE-Sephadex A-50 ${ }^{\mathrm{TM}}$ system, nearly $20 \%$ of G6PDdeficient samples were judged as normal. The red-colored hemoglobin in the reaction mixture together with the translucent DEAE-Sephadex A-50 ${ }^{\mathrm{TM}}$ particles may cause misdiagnosis. The proportion of the samples with normal activity that were diagnosed as 'normal' was $98 \%$ with DEAESephacel $^{\mathrm{TM}}$ and $100 \%$ with DEAE-Sephadex A- $50^{\mathrm{TM}}$.

The sensitivity and specificity in field tests are shown in Table 2. We used BFST as the standard because a previous study using the samples of genetically diagnosed G6PD-deficient patients showed that BFST had 100\% sensitivity in hemizygotes and $75 \%$ in heterozygotes and $100 \%$ specificity (Pujades et al., 1999). The sensitivity was significantly higher in males than in females when we use DEAE-Sephacel as anion-exchanger $(\mathrm{P}=0.04)$. Neither the sensitivity nor specificity differed significantly between DEAE-Sephadex $^{\mathrm{TM}}$ and DEAE-Sephacel ${ }^{\mathrm{TM}}$.

Comparison of field testing with laboratory assessment revealed that the sensitivity of field testing was lower than that of laboratory assessment. The P-value was 0.03 using DEAE-Sephadex A-50 and less than 0.001 using DEAESephacel. We found no difference in specificity. In field assessment, the sensitivity was significantly higher among males when we used DEAE-Sephacel $(\mathrm{p}=0.04)$. The actual value of the sensitivity was also high among males tested with DEAE-Sephacel, although there was no significant difference $(p=0.12)$. We suppose the discrepancy between laboratory and field assessments may be due to 3 reasons. One is the difference of genetic status between males and females. Because G6PD deficiency is an X-chromosomelinked abnormality, the enzyme activity is particularly vari-

Table 2. Sensitivity and specificity of the SSS using BFST as a standard

A. DEAE-Sephadex A-50

\begin{tabular}{cccc}
\hline \multirow{2}{*}{$\begin{array}{c}\text { Diagnosis } \\
\text { by BFST }\end{array}$} & \multicolumn{2}{c}{ Diagnosis by SSS (male/female) } & \multirow{2}{*}{$\begin{array}{c}\text { Total } \\
\text { (male/female) }\end{array}$} \\
\cline { 2 - 3 } G6PD deficiency & G6PD- normal & & G6iency \\
G6PD normal & $20(18 / 2)$ & $12(8 / 4)$ & \\
Total & $3(2 / 1)$ & $187(96 / 91)$ & $190(98 / 9)$ \\
\hline Totan & $23(20 / 3)$ & $199(104 / 95)$ & $222(124 / 98)$ \\
\hline
\end{tabular}

Sensitivity: male $69 \%(18 / 26)$, female $33 \%(2 / 6)$

Specificity:male $98 \%$ (96/98), female $99 \%$ (91/92)

B. DEAE-Sephacel

\begin{tabular}{|c|c|c|c|}
\hline \multirow{2}{*}{$\begin{array}{l}\text { Diagnosis } \\
\text { by BFST }\end{array}$} & \multicolumn{2}{|c|}{ Diagnosis by SSS (male/female) } & \multirow{2}{*}{$\begin{array}{c}\text { Total } \\
\text { (male/female) }\end{array}$} \\
\hline & G6PD deficiency & G6PD- normal & \\
\hline G6PD deficiency & $17(13 / 4)$ & $9(3 / 6)$ & $26(16 / 10)$ \\
\hline G6PD normal & $9(5 / 4)$ & $354(171 / 183)$ & $363(176 / 187)$ \\
\hline Total & $26(18 / 8)$ & $363(174 / 189)$ & $389(192 / 197)$ \\
\hline
\end{tabular}

Sensitivity: male $81 \%(13 / 16)$, female $40 \%(4 / 10)^{*}$

Specificity:male $97 \%$ (171/176), female 98\% (183/187)

*Significantly different $(\mathrm{P}=0.04)$ 
able in heterozygous females.

The crude G6PD level of blood may also be affected by anemia caused by various illnesses. Variable $\mathrm{Hb}$ levels together with various levels of G6PD activity in field samples could partially explain the discrepancy of these 2 tests, although our laboratory assessment showed that the G6PDdeficient samples could be distinguished from G6PDnormal samples.

We also consider it possible that the discrepancy between two tests may be due to inaccurate evaluation by SSS The definite cut-off level of coloring should be assessed by comparison of BFST and SSS using genetically confirmed samples.

Comparison of the results of SSS and BFST with the results of genetic analysis showed that 23 of 27 G6PDdeficient individuals detected by SSS, including two heterozygous females, had mutations (Table 3). All samples for which the results of SSS coincided with those of BFST were shown to have mutations, regardless of the anion exchanger used for the analysis. One case of class 2 G6PD mutation, $383 \mathrm{~T} \rightarrow \mathrm{C}$ (G6PD Vanua Lava), was detected among four cases that were diagnosed as 'G6PD-deficient' using SSS with DEAE-Sephacel ${ }^{\mathrm{TM}}$ but diagnosed as 'G6PD normal' with BFST. This result suggests that some cases of G6PD deficiency may be undetectable using BFST, although a study showed that the G6PD-deficient hemizygotes could be distinguished from heterozygotes and normal controls using BFST (Pujades et al ., 1999).

Including all reagents and disposable supplies, the estimated cost for one test of SSS with DEAE-Sephadex A$50^{\mathrm{TM}}$ was approximately half that of a test of SSS with the DEAE-Sephacel $^{\mathrm{TM}}$ (0.15 US dollar and 0.30 US dollar, respectively). The cost of both of these systems is below an acceptable limit.

SSS is a quick, simple and reliable screening test for G6PD deficiency, although a larger field study is necessary for more precise evaluation of SSS. All the procedures in this test could be completed in field conditions without any electric equipment except during some preparations prior to the study. Using either anion exchanger, it can be used for quick detection of G6PD deficiency in various situations including field surveys, mass-treatment of malaria and differ-

Table 3. The results of genetic analysis and the BFST of 'G6PD-deficient' samples diagnosed by SSS

\begin{tabular}{cccccc}
\hline Diagosis & \multicolumn{2}{c}{ DEAE-Sephadex A-50 } & & \multicolumn{2}{c}{ DEAE-Sephacel } \\
\cline { 2 - 3 } \cline { 5 - 6 } by BFST & Analyzed & Variants & & Analyzed & Variants \\
\hline Deficient & 17 & $17(1)$ & & 5 & $5(1)$ \\
Normal & 1 & 0 & & 4 & 1 \\
\hline Total & 18 & $17(1)$ & & 9 & $6(1)$ \\
\hline & & & & Heterozygotes in parentheses
\end{tabular}

ential diagnosis of hemolytic anemia, particularly in areas with substandard laboratory conditions. Although the whitecolored DEAE-Sephacel $^{\mathrm{TM}}$ gives better reaction visibility, the DEAE-Sephadex A-50 ${ }^{\mathrm{TM}}$ system is more cost-effective, and is therefore preferable financially, particularly for field studies.

\section{ACKNOWLEDGEMENTS}

The authors are indebted to Dr. Akira Hirono (Okinaka Memorial Institute for Medical Research) for valuable suggestions and critical reading of the manuscript. This study was supported by research grants from the Japanese Ministry of Education, Science, Culture and Sports (Grant No. 10041205 granted to Matsuoka, and 09041179 to Kawamoto). It was also supported by Toyota Foundation (Grant No. 96B3-011, to Kawamoto), the large-scale cooperative program (No. 2) from Japan Society for Promotion of Science (to Kawamoto), and a grant for International Health Cooperation Research from the Ministry of Health, Labour and Welfare (Grant No. 13-A-5, to Ishii).

\section{REFERENCES}

1) Beutler E: A series of new screening procedures for pyruvate kinase deficiency, glucose-6-phosphate dehydrogenase deficiency and glutathione reductase deficiency. Blood 28: 553-562, 1966.

2) Fairbanks, V.F., and Beutler, E. (1962): A simple method for detection of erythrocyte glucose-6-phosphate dehydrogenase deficiency (G6PD Spot Test). Blood, 20, 591601

3) Fujii, H., Takahashi, K. and Miwa, S. (1984): A new simple screening method for glucose-6-phosphate dehydrogenase deficiency. Acta Haematol. Jpn., 47, 185-188

4) Hirono, A., Fujii, H. and Miwa, S. (1998): An improved single-step screening method for glucose-6-phosphate dehydrogenase deficiency. Jpn. J. Trop. Med. Hyg., 26, $1-4$

5) Hirono, A., Miwa, S., Fujii, H., Ishida, F., Yamada, K. and Kubota, K. (1994): Molecular study of eight Japanese cases of glucose-6-phosphate dehydrogenase deficiency by nonradioisotopic single-strand conformation polymorphism analysis. Blood, 833, 3363-3368

6) Hirono, A., Fujii, H., Takano, T., Chiba, Y., Azuno, Y. and Miwa, S. (1997): Molecular analysis of eight biochemically unique glucose-6-phosphate dehydrogenase variants found in Japan. Blood 1997; 89: 4624-4627

7) Iwai, K., Hirono, A., Matsuoka, H., Kawamoto, F., Horie, T., Lin, K., Tantular, I.S., Dachlan, Y.P., Notopuro, H., Hidayah, N.I., Salim, A.M.A., Fujii, H., Miwa, S. and Ishii, A. (2001): Distribution of Glucose-6-phosphate dehydrogenase mutations in Southeast Asia. Hum. Genet. 2001; 108: 445-449 
8) Jansen G, Koenderman L, Rijksen G, Cats BP, Staal GEJ: Characteristics of hexokinase, pyruvate kinase, and glucose-6-phosphate dehydrogenase during adult and neonatal reticulocyte maturation. Am. J. Hematol. 20: 203-215, 1985.

9) Pujades, A., Lewis, M., Salvati, A.M., Miwa, S., Fujii, H., Zarza, R., Alvarez, R., Rull, E., and Corrons, J.L.V. (1999): Evaluation of the blue formazan spot test for screening glucose 6 phosphate dehydrogenase deficiency.
Int. J. Hematol., 69, 234-236

10) Tantular, I.S., Iwai, K., Lin, K., Basuki, S., Horie, T., Htay, H.H., Marwoto, H., Wongsrichanalai, C., Dachlan, Y.P., Kojima, S., Ishii, A. and Kawamoto, F. (1999): Field trials of a rapid test for G6PD deficiency in combination with a rapid diagnosis of malaria. Trop. Med. Int. Health, $4,245-250$

11) W.H.O. Working Group (1989): Glucose-6-phosphate dehydrogenase deficiency. Bull. W.H.O., 67, 601-611 